\title{
Dynamical analysis of the general beam model with singularity function
}

\author{
Duygu DÖNMEZ DEMIR*,*, B. Gültekin SINIR** and Emine KAHRAMAN* \\ *Department of Mathematics, Manisa Celal Bayar University, Manisa, Turkey \\ **Department of Civil Engineering, Manisa Celal Bayar University, Manisa, Turkey \\ *Corresponding Author: duygu.donmez@cbu.edu.tr
}

Submitted: 02/05/2019

Revised: $23 / 12 / 2020$

Accepted: $04 / 01 / 2021$

\begin{abstract}
The aim of this study is to present a general model with variable coefficients corresponding to some structural elements such as beam, string, bar, and rod. To solve general model with variable coefficients, a different solution procedure combining a method of multiple scales (MMS) and a finite difference method (FDM) is presented in this study. This technique provides an advantage in the numerical solution of the structural element model containing any discontinuity and in its dynamical analysis by perturbation method. Furthermore, two problems including discontinuity are considered to show the accuracy of the method presented. The comparisons of the numerical results obtained from the proposed method and classical method are introduced.
\end{abstract}

Keywords: General model; Method of multiple scales; Finite differences method; Singularity function; Discontinuous beams.

\section{INTRODUCTION}

Many engineering structures consist of the structural elements involving some discontinuities. The dynamic modelling of these structures leads to a set of partial differential equations (PDE). Generally, the mathematical models of this type do not contain the discontinuous functions such as Dirac delta and Heaviside step function; therefore, it can be solved analytically in a linear case. The same structures may be modeled by single differential equation instead of a set of equations when the system has discontinuities. In this case, a numerical method is needed in analyzing the model including singularity function.

Recently, Dinev (2012) has suggested an approach for analytical solution of the problem of bending a beam on an elastic foundation using singularity functions. Caddemi and Morassi (2013) proposed a verification of the rotational elastic spring model of an open crack formulated by suitable Dirac's Delta functions in a beam in bending deformation. Caddemi et al. (2013a) presented the closed-form solutions of the Timoshenko beam model subject to internal singularities leading to deflection and rotation discontinuities. Besides, a model of the stepped Timoshenko beam under deflection and rotation discontinuities that adopted Heaviside's and Dirac's Delta functions along the span was suggested by Caddemi et al. (2013b). For static and vibration analyses of stepped beam using singularity functions, Cheng et al. (2014) introduced a systematic approach, which is performed by directly differentiating the analytical deflection function with respect to any beam-related design variable.

In this study, a general model corresponding to the structural elements with different discontinuities is introduced. The general solution procedure is more effective instead of solving each problem, separately. For this purpose, many researchers (Lacarbonara, 1999, Hosseini \& Zamanian, 2013, Ghayesh et al., 2012) have introduced some general 
models with operator notations, which are suitable for perturbation calculations. A different approach is presented to solve the general model proposed in this study. This approach has the advantages of the numerical method in the discontinuity case, as well as the perturbation technique in the dynamical analysis. The mathematical model of the problem containing discontinuity can be derived in two different ways. First, the system is separated from each point including discontinuity, and the equation is separately written for each part. Thus, the number $M+1$ of equation is written, where the number of discontinuities equals $M$. The total number of boundary and transient conditions is $4 \times(M+1)$. Applying these conditions, the system of the linear algebraic equations is obtained as four times of span numbers. It is almost impossible to solve such a system for the large numbers $M$. Second, instead of a set of equations, the problem can be modeled by one equation including singularity function. Firstly, the MMS is directly applied to the equation having singularity function. At the first order, a linear equation is obtained. Substituting the assumption of first order into the equation yields an ordinary differential equation having singularity function. Since the solution of the differential equation with the singularity function is difficult to obtain analytically, numerical methods are needed. For this purpose, the finite difference method is used in this study.

\section{GOVERNING EQUATION}

The general structural dynamic model (Sınır, 2015) including harmonically internal and external excitations is

$$
\begin{aligned}
& a(x) \ddot{y}+L_{1}[y]+\varepsilon\left\{L_{2}[y] \cos \Omega_{1} t+F(x) \cos \Omega_{2} t+L_{3}[\dot{y}]\right\}=0 \\
& B_{11}[y]=B_{12}[y]=0 \text { and } B_{21}[y]=B_{22}[y]=0
\end{aligned}
$$

where $y(x, t)$ is the transverse deflection, $x$ is an axis, which denotes direction of the structure element, and $t$ is the time variable, and $F(x)$ is the amplitude of external force. $L_{2}[y] \cos \Omega_{1} t$ and $F(x) \cos \Omega_{2} t$ correspond to parametric excitation and harmonically external force, respectively. $\Omega_{1}$ and $\Omega_{2}$ are the frequencies of the internal and external excitation, respectively. $\varepsilon$ is a small dimensionless parameter. The dot represents differentiation with respect to time $t$. The subscripts of $i, j$ at boundary conditions represent $j$ th condition of the $i$ th support for $B_{i j}$. $a(x)$ is the arbitrary function representing the variation of the mass and cross section. $L_{1}$ is related to stiffness structural elements. $L_{3}$ represents viscoelastic properties of the system. $a(x)$ and the operator $L_{1}$ may contain the singularity function to define discontinuities of one-dimensional structure (such as crack, stepped beam, or multisupport). $L_{1}, L_{2}$ and $L_{3}$ are linear and self-adjoint differential operators having variable coefficients. The differential operator $L$ having variable coefficients can be demonstrated as

$$
L=p_{4}(x) \frac{d^{4}}{d x^{4}}+p_{3}(x) \frac{d^{3}}{d x^{3}}+p_{2}(x) \frac{d^{2}}{d x^{2}}+p_{1}(x) \frac{d}{d x}+p_{0}(x)
$$

where the highest derivative is denoted by fourth order since the mathematical model of the beams as structural elements is considered $\left(L_{1}, L_{2}\right.$ and $L_{3}$ can be in form of the operator $\left.L\right)$. The space domain is considered as [0,1]. Also, these linear operators correspond to the other one-dimensional structural element such as bar, string, and cable. For example, $p_{3}$ and $p_{4}$ vanish for a bar or string structure element.

\section{METHOD OF MULTIPLE SCALES}

In this section, MMS is directly applied to the governing equation. Perturbation series expansion is assumed as

$$
y\left(x, \mathrm{~T}_{0}, \mathrm{~T}_{1} ; \varepsilon\right)=y_{0}\left(x, \mathrm{~T}_{0}, \mathrm{~T}_{1} ; \varepsilon\right)+\varepsilon y_{1}\left(x, \mathrm{~T}_{0}, \mathrm{~T}_{1} ; \varepsilon\right)+\ldots
$$

where $\mathrm{T}_{n}$ is different time scales in form of $\mathrm{T}_{n}=\varepsilon^{n} t$. The time derivatives in terms of the new time scales are defined by 


$$
\frac{d}{d t}=D_{0}+\varepsilon D_{1}+\cdots \text { and } \frac{d^{2}}{d t^{2}}=D_{0}^{2}+2 \varepsilon D_{0} D_{1}+\cdots
$$

where $D_{i}=\partial / \partial T_{i}$. Substituting Eqs. (3-4) into Eq. (1-2) and separating terms at each order $\varepsilon$ yield

$$
\begin{aligned}
& O(1): a(x) D_{0}^{2} y_{0}+L_{1}\left[y_{0}\right]=0 \\
& B_{11}\left[y_{0}\right]=B_{12}\left[y_{0}\right]=0 \text { and } B_{21}\left[y_{0}\right]=B_{22}\left[y_{0}\right]=0 \\
& O(\varepsilon): a(x) D_{0}^{2} y_{1}+L_{1}\left[y_{1}\right]=-L_{3}\left[D_{0} y_{0}\right]-2 a(x) D_{0} D_{1} y_{0}-L_{2}\left[y_{0}\right] \cos \Omega_{1} T_{0} \\
& -F(x) \cos \Omega_{2} T_{0} \\
& B_{11}\left[y_{1}\right]=B_{12}\left[y_{1}\right]=0 \text { and } B_{21}\left[y_{1}\right]=B_{22}\left[y_{1}\right]=0
\end{aligned}
$$

The solution at first order is assumed as

$y_{0}\left(x, \mathrm{~T}_{0}, \mathrm{~T}_{1}\right)=\left(A_{n}\left(\mathrm{~T}_{1}\right) e^{i \omega_{n} \mathrm{~T}_{0}}+\bar{A}_{n}\left(\mathrm{~T}_{1}\right) e^{-i \omega_{n} \mathrm{~T}_{0}}\right) X_{n}(x) ; n=1,2,3, \ldots$

where $A_{n}$ is the complex amplitude. Substituting Eq. (9) into Eq. (5), the relation in the following is obtained.

$$
L_{1}\left[X_{n}\right]-a(x) \omega_{n}^{2} X_{n}=0
$$

Eq. (10) can be called as an eigenvalue-eigenfunction problem. $\omega_{n}$ represents the eigenvalues, and $X_{n}$ corresponds to eigenfunctions of the system. From Eqs. (10), $X_{n}$ and $\omega_{n}$ can be calculated. Since the resulting equation is the ordinary differential equation with variable coefficient, finite differences method is used for the approximate solution.

\section{FINITE DIFFERENCES METHOD}

For finite differences method, there are three different finite differences schemes: forward differences, backward differences, and central differences. For small truncation error, the central difference is chosen. Then, first four derivatives are given as follows:

$$
\begin{gathered}
X_{j}^{\prime} \approx \frac{X_{j+1}-X_{j-1}}{2 \Delta x} \text { and } X_{j}^{\prime \prime} \approx \frac{X_{j+1}-2 X_{j}+X_{j-1}}{\Delta x^{2}} \\
X_{j}^{\prime \prime \prime}=\frac{X_{j+2}-2 X_{j+1}+2 X_{j-1}-X_{j-2}}{2 \Delta x^{3}} \text { and } X_{j}^{i v}=\frac{X_{j+2}-4 X_{j+1}+6 X_{j}-4 X_{j-1}+X_{j-2}}{\Delta x^{4}}
\end{gathered}
$$

where $\Delta x=1 / N . N$ is the total number of short segments into system. In these discretized forms, the subscript indicates spatial node. Substituting Eq. (11) into Eq. (10) yields the discretized equation at jth spatial node as

$$
b_{4, j} X_{n, j+2}+b_{3, j} X_{n, j+1}+b_{2, j} X_{n, j}+b_{1, j} X_{n, j-1}+b_{0, j} X_{n, j-2}=0
$$

where

$$
\begin{aligned}
& b_{0, j}=\frac{p_{4, j}}{\Delta x^{4}}-\frac{p_{3, j}}{2 \Delta x^{3}}, b_{1, j}=-\frac{4 p_{4, j}}{\Delta x^{4}}+\frac{p_{3, j}}{\Delta x^{3}}+\frac{p_{2, j}}{\Delta x^{2}}-\frac{p_{1, j}}{2 \Delta x}, b_{2, j}=\frac{6 p_{4, j}}{\Delta x^{4}}-\frac{2 p_{2, j}}{\Delta x^{2}}+p_{0, j} \\
& b_{3, j}=-\frac{4 p_{4, j}}{\Delta x^{4}}-\frac{p_{3, j}}{\Delta x^{3}}+\frac{p_{2, j}}{\Delta x^{2}}+\frac{p_{1, j}}{2 \Delta x}, b_{4, j}=\frac{p_{4, j}}{\Delta x^{4}}+\frac{p_{3, j}}{2 \Delta x^{3}}
\end{aligned}
$$


The boundary conditions are

$$
\begin{aligned}
& B_{11}\left(X_{n}\right)=X_{n}, B_{12}\left(X_{n}\right)=X_{n}^{\prime \prime}, B_{21}\left(X_{n}\right)=X_{n}, B_{22}\left(X_{n}\right)=X_{n}^{\prime \prime} \\
& B_{11}\left(X_{n}\right)=X_{n}, B_{12}\left(X_{n}\right)=X_{n}^{\prime}, B_{21}\left(X_{n}\right)=X_{n}, B_{22}\left(X_{n}\right)=X_{n}^{\prime}, \\
& B_{11}\left(X_{n}\right)=X_{n}, B_{12}\left(X_{n}\right)=X_{n}^{\prime \prime}, B_{21}\left(X_{n}\right)=X_{n}, B_{22}\left(X_{n}\right)=X_{n}^{\prime}
\end{aligned}
$$

for pinned-pinned (PP) support, clamped-clamped (CC) support, and pinned-clamped (PC) support, respectively. Using boundary conditions, Eq. (12) yields the algebraic equation system. In this study, the algebraic equations system in matrix form is given for PP, CC, and PC support conditions. Finite differences applications of the boundary conditions are as follows:

$$
\begin{aligned}
& X_{n, 0}=0, X_{n,-1}=-X_{n, 1}, X_{n, N}=0, X_{n, N+1}=-X_{n, N-1} \\
& X_{n, 0}=0, X_{n,-1}=X_{n, 1}, X_{n, N}=0, X_{n, N+1}=X_{n, N-1} \\
& X_{n, 0}=0, X_{n,-1}=-X_{n, 1}, X_{n, N}=0, X_{n, N+1}=X_{n, N-1}
\end{aligned}
$$

for PP, CC, and PC support, respectively. Using the boundary conditions (17) and the relations (12-13), the algebraic equation system can be written in the following matrix form:

$$
\left[\begin{array}{cccccccccccccc}
b_{2,1}-b_{0,1} & b_{3,1} & b_{4,1} & 0 & 0 & 0 & \ldots & 0 & 0 & 0 & 0 & 0 & 0 \\
b_{1,2} & b_{2,2} & b_{3,2} & b_{4,2} & 0 & 0 & \ldots & 0 & 0 & 0 & 0 & 0 & 0 \\
b_{0,3} & b_{1,3} & b_{2,3} & b_{3,3} & b_{4,3} & 0 & \ldots & 0 & 0 & 0 & 0 & 0 & 0 \\
0 & b_{0,4} & b_{1,4} & b_{2,4} & b_{3,4} & b_{4,4} & \ldots & 0 & 0 & 0 & 0 & 0 & 0 \\
\vdots & \vdots & \vdots & \vdots & \vdots & \vdots & \ddots & \vdots & \vdots & \vdots & \vdots & \vdots & \vdots \\
0 & 0 & 0 & 0 & 0 & 0 & \ldots & b_{0, N-4} & b_{1, N-4} & b_{2, N-4} & b_{3, N-4} & b_{4, N-4} & 0 \\
0 & 0 & 0 & 0 & 0 & 0 & \ldots & 0 & b_{0, N-3} & b_{1, N-3} & b_{2, N-3} & b_{3, N-3} & b_{4, N-3} \\
0 & 0 & 0 & 0 & 0 & 0 & \ldots & 0 & 0 & b_{0, N-2} & b_{1, N-2} & b_{2, N-2} & b_{3, N-2} \\
0 & 0 & 0 & 0 & 0 & 0 & \ldots & 0 & 0 & 0 & b_{0, N-1} & b_{1, N-1} & b_{2, N-1}-b_{4, N-1}
\end{array}\right]\left[\begin{array}{c}
X_{n, 1} \\
X_{n, 2} \\
X_{n, 3} \\
X_{n, 4} \\
\vdots \\
X_{n, N-4} \\
X_{n, N-3} \\
X_{n, N-2} \\
X_{n, N-1}
\end{array}\right]=\left[\begin{array}{c}
0 \\
0 \\
0 \\
0 \\
\vdots \\
0 \\
0 \\
0 \\
0
\end{array}\right]
$$

Similarly, the other algebraic equation systems for CC and PC can be obtained. The determinant of the matrices of the coefficients (20) must be equal to zero for nontrivial solutions. The mode shapes $X_{n}$ are obtained depending on the node $N / 2$. Thus, $y_{0}$ is determined from the solution of Eq. (5). For the solution of $\varepsilon$-order, substituting Eq. (9) into Eq. (7) yields

$$
\begin{aligned}
a(x) D_{0}^{2} y_{1}+L_{1}\left[y_{1}\right]= & -\left(2 a(x) i \omega_{n} X_{n} D_{1} A_{n}+i \omega_{n} A_{n} L_{3}\left[X_{n}\right]\right) e^{i \omega_{n} T_{0}} \\
& -\frac{L_{2}\left[X_{n}\right]}{2}\left[A_{n}\left(e^{i\left(\omega_{n}+\Omega_{1}\right) T_{0}}+e^{i\left(\omega_{n}-\Omega_{1}\right) T_{0}}\right)\right]-\frac{F(x)}{2} e^{i \Omega_{2} T_{0}}+c c
\end{aligned}
$$

where $c c$ denotes complex conjugates. The solution of Eq. (21) can be considered as

$$
y_{1}\left(x, T_{0}, T_{1}\right)=\varphi_{n}\left(x, T_{1}\right) e^{i \omega_{n} T_{0}}+Y\left(x, T_{0}, T_{1}\right)+c c
$$

where the first and second terms are related to secular and nonsecular terms, respectively. The term related to force is either secular or nonsecular term depending on its frequency. 


\section{CASE STUDIES}

Applying solvability conditions (Nayfeh, 1981), some definite integrals including mode shapes $X_{n}$ and its derivatives are obtained in the form of

$$
\alpha_{i n}=\int_{0}^{1} X_{n} L_{i}\left[X_{n}\right] d x
$$

where only numerical values of $X_{n}$ exist. In the approximate calculation of integrals from the solvability condition, Simpson's method, one of the numerical integration rules, was used. The formula of Simpson's method is given as

$$
\int_{0}^{1} f(x) d x \cong \frac{\Delta x}{3}\left[f_{0}+4\left(f_{1}+f_{3}+\ldots+f_{2 N-1}\right)+2\left(f_{2}+f_{4}+\ldots+f_{2 N}\right)+f_{N}\right]
$$

where $N$ is the number of subintervals, $\Delta x$ is the step length $(\Delta x=1 / N)$, and the function $f_{j}$ corresponds to

$$
\begin{aligned}
f_{j}=\frac{X_{n, j}}{\Delta x^{4}}[ & X_{n, j+2}\left(\Delta x p_{3}\left(x_{j}\right)+p_{4}\left(x_{j}\right)\right)+X_{n, j+1}\left(\Delta x^{3} p_{1}\left(x_{j}\right)+\Delta x^{2} p_{2}\left(x_{j}\right)\right. \\
& \left.-2 \Delta x p_{3}\left(x_{j}\right)-4 P_{4}\left(x_{j}\right)\right)+X_{n, j}\left(\Delta x^{4} p_{0}\left(x_{j}\right)-2 \Delta x^{2} p_{2}\left(x_{j}\right)+6 p_{4}\left(x_{j}\right)\right) \\
& +X_{n, j-1}\left(-\Delta x^{3} p_{1}\left(x_{j}\right)+\Delta x^{2} p_{2}\left(x_{j}\right)+2 \Delta x p_{3}\left(x_{j}\right)-4 p_{4}\left(x_{j}\right)\right) \\
& \left.+X_{n, j-2}\left(-\Delta x p_{3}\left(x_{j}\right)+p_{4}\left(x_{j}\right)\right)\right]
\end{aligned}
$$

\section{$\Omega_{1}$ AWAY FROM 0 AND $2 \omega_{n}, \Omega_{2}$ AWAY FROM $\omega_{n}$}

Substituting Eq. (9) into Eq. (7) and eliminating the terms producing secularity yield the equation in the following:

$$
L_{1}\left(\varphi_{n}\right)-a(x) \omega_{n}^{2} \varphi_{n}=-2 a(x) i \omega_{n} X_{n} D_{1} A_{n}-i \omega_{n} L_{3}\left(X_{n}\right) A_{n}
$$

The solvability condition requires (see reference (Nayfeh, 1981) for calculating in detailed)

$$
D_{1} A_{n}+\alpha_{3 n} A_{n}=0
$$

where the normalization is $\int_{0}^{1} a(x) X_{n}^{2} d x=1$, and the coefficient $\alpha_{3 n}$ is $\alpha_{3 n}=\frac{1}{2} \int_{0}^{1} X_{n} L_{3}\left[X_{n}\right] d x$.

Then, $A_{n}$ is obtained from Eq. (27) as follows:

$$
A_{n}=C e^{-\alpha_{3 n} T_{1}}
$$

where $C$ is an arbitrary constant, and $\alpha_{3 n}$ is always real and positive. Then, the amplitude of the system exponentially decreases, and the solution is stable.

\section{$\Omega_{1}$ CLOSE TO $0, \Omega_{2}$ AWAY FROM $\omega_{n}$}

In this case, $\Omega_{1} \approx 0$ and $\Omega_{2} \neq \omega_{n}$ are assumed, where the detuning parameter $\sigma$ is used to describe the nearness $\Omega_{1}$ to 0 . Then, $\Omega_{1}=\varepsilon \sigma$. Similarly, the solvability condition is obtained as follows:

$D_{1} A_{n}+\left(\alpha_{3 n}+2 i \alpha_{2 n} \cos \sigma T_{1}\right) A_{n}=0$

where $\alpha_{2 n}=-\frac{1}{4 \omega_{n}} \int_{0}^{1} X_{n} L_{2}\left[X_{n}\right] d x$. From the solution of Eq. (29), the amplitude is found as 


$$
A_{n}=A_{0} e^{-\alpha_{3 n} T_{1}-\frac{2 i \alpha_{2 n}}{\sigma} \sin \sigma T_{1}}
$$

The term $A_{0} e^{-\alpha_{3 n} T_{1}}$ leads to diminish the amplitude. Since $\left|\sin \sigma T_{1}\right| \leq 1$, the complex amplitudes are bounded in time. However, this order of approximation has not got any instability.

\section{$\Omega_{1}$ CLOSED TO $2 \omega_{n}, \Omega_{2}$ AWAY FROM $\omega_{n}$}

The excitation frequency has the variation around two times of the natural frequencies such that $\Omega_{1}=2 \omega_{n}+\varepsilon \sigma$, where $\sigma$ is the detuning parameter. Thus, one obtains

$D_{1} A_{n}+\alpha_{3 n} A_{n}+i \alpha_{2 n} e^{i \sigma T_{1}} \bar{A}_{n}=0$.

The polar form of $A_{n}$ is assumed as

$A_{n}=\frac{1}{2} a_{n}\left(T_{1}\right) e^{i \beta_{n}\left(T_{1}\right)}$

Substituting Eq. (32) into Eq. (31) and separating real and imaginary parts of the resulting equation yield

$$
\begin{aligned}
& \frac{d a_{n}}{d T_{1}}+\alpha_{3 n} a_{n}-a_{n} \alpha_{2 n I} \sin \gamma=0 \\
& \sigma-\frac{d \gamma}{d T_{1}}+\alpha_{2 n I} \cos \gamma=0
\end{aligned}
$$

where $\gamma=\sigma T_{1}-2 \beta$. Since $d a_{n} / d T_{1}$ and $d \gamma / d T_{1}$ should be equal to zero for steady state solutions, the detuning parameter is obtained as

$$
\sigma=\mp \sqrt{\alpha_{2 n I}^{2}-\alpha_{3 n}^{2}}
$$

\section{$\Omega_{2}$ CLOSED TO $\omega_{n}, \Omega_{1}$ AWAY FROM $2 \omega_{n}$ AND 0}

For $\Omega_{2}=\omega_{n}+\varepsilon \sigma$, the amplitude equation is obtained as follows:

$D_{1} A_{n}+\alpha_{3 n} A_{n}=\frac{1}{2} i \alpha_{1 n} e^{i \sigma T_{1}}$

where $\alpha_{1 n}=\frac{1}{2 \omega_{n}} \int_{0}^{1} F(x) X_{n} d x$. Substituting Eq. (32) into Eq. (36) and separating real and imaginary parts, the resulting equation is

$$
\begin{gathered}
\frac{d a_{n}}{d T_{1}}+\alpha_{3 n} a_{n}=-\alpha_{1 n I} \sin \gamma \\
\sigma a_{n}-\frac{d \gamma}{d T_{1}} a_{n}=\alpha_{1 n I} \cos \gamma
\end{gathered}
$$

where $\gamma=\sigma T_{1}-\beta$. Thus, $\sigma$ is found as

$$
\sigma=\mp \frac{1}{a_{n}} \sqrt{\alpha_{1 n I}^{2}-a_{n}^{2} \alpha_{3 n}^{2}}
$$

considering $d a_{n} / d T_{1}$ and $d \gamma / d T_{1}$ equal to zero for the steady state solutions. 


\section{BEAMS WITH MULTIPLE SPAN UNDER VERTICAL SPRING-SUPPORT SUBJECTED TO PARAMETRIC AXIAL FORCE}

In this section, the beam having linear elastic spring at internal support (Sinır \& Sinır, 2011) is considered. The equation of motion involving a singularity function for the number $M$ of linear elastic spring is presented as follows:

$$
E I \hat{y}^{i v}+\hat{P} \hat{y}^{\prime \prime}+\sum_{j=1}^{M} \hat{k}_{j} \delta\left(\hat{x}-\hat{\eta}_{j}\right) \hat{y}+m \ddot{\hat{y}}+\hat{\mu} \dot{\hat{y}}=0 ; j=1,2, \ldots, M
$$

and boundary conditions

$$
\hat{y}(0, \hat{t})=\hat{y}^{\prime \prime}(0, \hat{t})=0 \text { and } \hat{y}(1, \hat{t})=\hat{y}^{\prime \prime}(1, \hat{t})=0
$$

where $E$ and $I$ are the modulus of elasticity and the moment of inertia, respectively. $m$ denotes the mass per unit length. $\hat{\mu}$ is the linear viscous damping coefficient. $L$ describes the distance apart between two simple supports. $\hat{P}(\hat{t})$ is axially harmonic compressed by a loading such that $\hat{P}=\hat{P}_{0}+\varepsilon \hat{P}_{1} \cos \Omega \hat{t}$, where $\varepsilon$ is a small dimensionless parameter. $\hat{k}$ represents the spring constant. $\delta$ denotes Dirac delta function corresponding to singularity function $\left\langle\hat{x}-\hat{\eta}_{j}\right\rangle^{-1}$ (Lect. 12). The singularity functions are used to calculate deflections of beams with various loading and support conditions. Introducing the dimensionless terms for Eq. (40-41)

$$
y=\frac{\hat{y}}{L}, x=\frac{\hat{x}}{L}, t=\frac{\hat{t}}{L^{2}} \sqrt{\frac{E I}{m}} \text { and } \eta_{j}=\frac{\hat{\eta}_{j}}{L}, k_{j}=\frac{\hat{k}_{j} L^{3}}{E I}, P=\frac{\hat{P} L^{2}}{E I}, \mu=\frac{\hat{\mu} L^{2}}{\sqrt{E I m}},
$$

the resulting equation is obtained as

$$
\begin{aligned}
& y^{i v}+P y^{\prime \prime}+\sum_{j=1}^{M} k_{j} \delta\left(x-\eta_{j}\right) y+\ddot{y}+\mu \dot{y}=0 \\
& y(0, t)=y^{\prime \prime}(0, t)=0 \text { and } y(1, t)=y^{\prime \prime}(1, t)=0 .
\end{aligned}
$$

In this section, the dynamic response of elastic beam with one spring is analyzed for two spans. For $M=1$, the resulting equation (43) becomes

$$
y^{i v}+P y^{\prime \prime}+k \delta(x-\eta) y+\ddot{y}+\mu \dot{y}=0
$$

Thus, the operators given in the general model are as follows:

$$
L_{1}[y]=y^{i v}+P_{0} y^{\prime \prime}+k \delta(x-\eta) y, L_{2}[y]=P_{1} y^{\prime \prime}, L_{3}[\dot{y}]=\mu \dot{y}, a(x)=1, \Omega_{1}=\Omega
$$

Substituting these terms to Eq. (10), one obtains

$$
\begin{aligned}
& X_{n}^{i v}+P_{0} X_{n}^{\prime \prime}+k X_{n} \delta(x-\eta)-\omega_{n}^{2} X_{n}=0 \\
& X_{n}(0)=X_{n}^{\prime \prime}(0)=0 \text { and } X_{n}(1)=X_{n}^{\prime \prime}(1)=0
\end{aligned}
$$

Then, the coefficients (13) according to Eq. (47) are as follows:

$$
\begin{aligned}
& b_{0, j}=1 / \Delta x^{4}, b_{1, j}=\left(P_{0} \Delta x^{2}-4\right) / \Delta x^{4}, b_{2, j}=\left[6-2 P_{0} \Delta x^{2}+\left(k \delta(x-\eta)-\omega_{n}^{2}\right) \Delta x^{4}\right] / \Delta x^{4} \\
& b_{3, j}=\left(P_{0} \Delta x^{2}-4\right) / \Delta x^{4}, b_{4, j}=1 / \Delta x^{4} .
\end{aligned}
$$


Here, all values except $b_{2, j}$ are constant for each the value of $j$. We substitute the coefficients $(49,50)$ into the matrix (20). For nontrivial solution, the determinant of matrix of the coefficients should be equal to zero. Using this condition, the natural frequency of the system can be approximately found. The mode shape is also numerically obtained. Proceeding the perturbative solution, cases 1,2, and 3 reveal this problem. Then, the coefficients $\alpha_{3 n}$ and $\alpha_{2 n}$ become

$$
\alpha_{3 n}=\frac{\mu}{2} \text { and } \alpha_{2 n}=-\frac{P_{1}}{4 \omega_{n}} \int_{0}^{1} X_{n} X_{n}^{\prime \prime} d x
$$

For calculating definite integral in the coefficient $\alpha_{2 n}$, Simpson's method is used. Then, the function $f_{j}$ in the integral (51) is

$$
f_{j}=\frac{X_{n, j}}{\Delta x^{2}}\left(X_{n, j+1}-2 X_{n, j}+X_{n, j-1}\right) .
$$

Comparing the obtained results, the same problem can be written as a set of equations of motions. Writing the equation in this form yields

$$
\begin{aligned}
& y_{1}^{i v}+P y_{1}^{\prime \prime}+\ddot{y}_{1}+\mu \dot{y}_{1}=0 \\
& y_{2}^{i v}+P y_{2}^{\prime \prime}+\ddot{y}_{2}+\mu \dot{y}_{2}=0
\end{aligned}
$$

with the boundary conditions $y_{1}(0, t)=y_{1}^{\prime \prime}(0, t)=0$ and $y_{2}(1, t)=y_{2}^{\prime \prime}(1, t)=0$ and the transient conditions $y_{1}=y_{2}, y_{1}^{\prime}=y_{2}^{\prime}, y_{1}^{\prime \prime}=y_{2}^{\prime \prime}$ and $y_{1}^{\prime \prime \prime}+P y_{1}^{\prime}-k y_{1}=y_{2}^{\prime \prime \prime}+P y_{2}^{\prime}$.

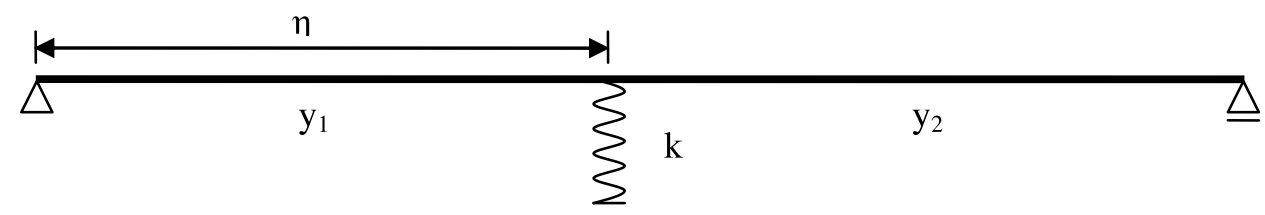

Figure 1. The elastic beam with one spring and two spans.

In Sinır \& Sinır (2011), the coefficient $\alpha_{2 n}$ is calculated as

$$
\alpha_{2 n}=-i \frac{P_{1}}{4 \omega_{n}} \int_{0}^{\eta} X_{1 n} X_{1 n}^{\prime \prime} d x+\int_{\eta}^{1} X_{2 n} X_{2 n}^{\prime \prime} d x .
$$

The comparison of the values of critical axially loading obtained for the coefficient of spring $k$ and the location of spring $\eta$ is given in the Table 1, where $N$ denotes the number of grid points in the expansion of finite differences. Similarly, the values of the natural frequencies for the various locations of spring are given in Table 2 .

Table 1. The comparison of axially critical loading obtained with Sinır \& Sinır (2011) and the present method (bold) for $N=200$.

\begin{tabular}{|c|c|c|c|c|}
\hline$k$ & \multicolumn{2}{|c|}{$\eta=100$} & \multicolumn{2}{c|}{$\eta=1000$} \\
\hline 0.1 & 11.6355 & $\mathbf{1 1 . 6 3 5 3}$ & 18.6836 & $\mathbf{1 8 . 6 8 2 0}$ \\
\hline 0.3 & 20.4587 & $\mathbf{2 0 . 4 5 8 2}$ & 30.7234 & $\mathbf{3 0 . 7 2 0 7}$ \\
\hline 0.5 & 29.2960 & $\mathbf{2 9 . 2 9 5 7}$ & 39.4784 & $\mathbf{3 9 . 4 7 5 2}$ \\
\hline
\end{tabular}


Table 2. The comparison of the natural frequencies obtained with Sinır \& Sinır (2011) and the present method (bold) for $P_{0}=10$ and $N=200$.

\begin{tabular}{|c|c|c|c|c|}
\hline$k$ & \multicolumn{2}{|c|}{$\eta=100$} & \multicolumn{2}{c|}{$\eta=1000$} \\
\hline 0.1 & 4.0239 & $\mathbf{4 . 0 2 3 6}$ & 9.7712 & $\mathbf{9 . 7 7 0 2}$ \\
\hline 0.3 & 10.4605 & $\mathbf{1 0 . 4 6 0 2}$ & 19.5262 & $\mathbf{1 9 . 5 2 4 4}$ \\
\hline 0.5 & 13.8591 & $\mathbf{1 3 . 8 5 8 7}$ & 34.1140 & $\mathbf{3 4 . 1 1 0 7}$ \\
\hline
\end{tabular}

Table 3. The comparison of the coefficients $\alpha_{2 n}$ obtained with Sinır \& Sinır (2011) and the present method (bold) for $P_{0}=10, P_{1}=1$ and $N=200$.

\begin{tabular}{|c|c|c|c|c|}
\hline$k$ & \multicolumn{2}{|c|}{$\eta=100$} & \multicolumn{2}{c|}{$\eta=1000$} \\
\hline 0.1 & -0.6150 & $\mathbf{- 0 . 6 1 5 0}$ & -0.2778 & $\mathbf{- 0 . 2 7 6 9}$ \\
\hline 0.3 & -0.2466 & $\mathbf{- 0 . 2 4 6 7}$ & -0.2212 & $\mathbf{- 0 . 2 2 9 5}$ \\
\hline 0.5 & -0.1792 & $\mathbf{- 0 . 1 7 9 2}$ & -0.0984 & $\mathbf{- 0 . 0 9 6 7}$ \\
\hline
\end{tabular}

Table 3 shows that the results obtained with classical method and finite differences are close to each other. It is seen that the numerical results and analytical solutions are almost the same.

\section{THE BEAM-MASS SYSTEM}

A beam-mass system consists of the Euler-Bernoulli beam and a concentrated mass on this beam. The equation of motion (Özkaya et al., 1997) according to proposed technique can be written as follows:

$$
\begin{aligned}
& {\left[\rho A+\bar{M}_{j} \delta\left(\hat{x}-x_{j}\right)\right] \ddot{\hat{w}}+E I \hat{w}^{i v}+\hat{\mu} \dot{\hat{w}}-\hat{g}(\hat{x}) \cos \hat{\Omega} \hat{t}=0 ; j=1,2, \ldots, n} \\
& \hat{w}(0, \hat{t})=\hat{w}^{\prime \prime}(0, \hat{t})=0 \text { and } \hat{w}(1, \hat{t})=\hat{w}^{\prime \prime}(1, \hat{t})=0 .
\end{aligned}
$$

where $\rho$ is the density, and $A$ is the cross-sectional area. $\bar{M}_{j}$ represents the concentrated mass. $\hat{\mu}, \hat{g}$ and $\hat{\Omega}$ denote viscous damping coefficient, the external excitation amplitude, and frequency. Introducing the dimensionless parameters

$$
\begin{aligned}
& w=\hat{w} / L, x=\hat{x} / L, \eta_{j}=x_{j} / L, t=\left(\hat{t} / L^{2}\right) \sqrt{E I / \rho A} \\
& \alpha_{j}=\bar{M}_{j} / \rho A L, \Omega=\hat{\Omega} L^{2} / \sqrt{E I / \rho A}, \varepsilon g=\hat{g} L^{3} / E I, \varepsilon \mu=\hat{\mu} L^{2} / \sqrt{E I \rho A},
\end{aligned}
$$

then, the resulting equation is

$$
\begin{aligned}
& {\left[1+\alpha_{j} \delta\left(x-\eta_{j}\right)\right] \ddot{w}+w^{i v}+\mu \dot{w}-g(x) \cos \Omega t=0} \\
& w(0, t)=w^{\prime \prime}(0, t)=0 \text { and } w(1, t)=w^{\prime \prime}(1, t)=0 .
\end{aligned}
$$

where $\alpha$ is the ratio of the concentrated mass to the beam-mass. The operators corresponding to the general model are

$$
L_{1}[y]=w^{i \nu}, L_{2}[y]=0, L_{3}[\dot{y}]=\mu \dot{w}, a(x)=1+\alpha_{j} \delta\left(x-\eta_{j}\right), \Omega_{2}=\Omega, F(x)=-g(x)
$$


Then, Eq. (10) is obtained as

$$
\begin{aligned}
& X_{n}^{i v}-\left(1+\alpha_{j} \delta\left(x-\eta_{j}\right)\right) \omega_{n}^{2} X_{n}=0 \\
& X_{n}(0)=X_{n}^{\prime \prime}(0)=0 \text { and } X_{n}(1)=X_{n}^{\prime \prime}(1)=0 .
\end{aligned}
$$

The coefficients (13) from Eq. (63) are obtained as follows:

$$
\begin{aligned}
& b_{0, j}=1 / \Delta x^{4}, b_{1, j}=-4 / \Delta x^{4}, b_{2, j}=\left[6-\omega_{n}^{2}\left(1+\alpha_{j} \delta\left(x-\eta_{j}\right)\right) \Delta x^{4}\right] / \Delta x^{4} \\
& b_{3, j}=-4 / \Delta x^{4}, b_{4, j}=1 / \Delta x^{4}
\end{aligned}
$$

Substituting the coefficients (63) to the matrix (20), the solution of the resulting matrix is smoothly obtained. Thus, the natural frequency of the system and the mode shape can be calculated. Cases 1 and 4 appear in this problem from the perturbative solution. Then, the coefficients $\alpha_{3 n}$ and $\alpha_{1 n}$ are as follows:

$$
\alpha_{3 n}=\frac{\mu}{2} \text { and } \alpha_{1 n}=-\frac{1}{2 \omega_{n}} \int_{0}^{1} g(x) X_{n} d x
$$

The linear form of the mathematical models in (Özkaya et al., 1997 \& Özkaya, 2001) is similar to the proposed equation in this study. In the result of performed calculations, the numerical comparisons are given in the following.

Table 4. The first three natural frequencies for different mass ratios and mass locations for one mass, where $N=100$.

\begin{tabular}{cccccccc}
\hline$\alpha$ & $\eta$ & $\omega_{1}$ & Özk.97 & $\omega_{2}$ & Özk.97 & $\omega_{3}$ & Özk.97 \\
\hline \multirow{2}{*}{1} & 0.0 & 9.8688 & 9.8695 & 39.4654 & 39.4784 & 88.7607 & 88.8264 \\
& 0.1 & 8.9954 & 8.9962 & 29.8755 & 29.8891 & 66.0088 & 66.0691 \\
& 0.2 & 7.4533 & 7.4541 & 26.9359 & 26.9462 & 73.4569 & 73.5140 \\
& 0.3 & 6.3941 & 6.3946 & 29.7397 & 29.7503 & 86.6638 & 86.7293 \\
& 0.4 & 5.8463 & 5.8468 & 35.2250 & 35.2374 & 79.9135 & 79.9788 \\
& 0.5 & 5.6791 & 5.6795 & 39.4654 & 39.4784 & 67.8305 & 67.8883 \\
& & & & & & & \\
& 0.0 & 9.8688 & 9.8695 & 39.4654 & 39.4785 & 88.7607 & 88.8264 \\
& 0.1 & 5.3312 & 5.3322 & 19.8249 & 19.8959 & 59.0482 & 59.0995 \\
& 0.2 & 3.2594 & 3.2598 & 22.0495 & 22.0545 & 70.7174 & 70.7723 \\
& 0.3 & 2.5276 & 2.5279 & 26.7608 & 26.7706 & 86.0802 & 86.1462 \\
& 0.4 & 2.2250 & 2.2252 & 33.6682 & 33.6806 & 77.2029 & 77.2690 \\
& 0.5 & 2.1393 & 2.1395 & 39.4654 & 39.4785 & 62.3954 & 62.4517 \\
\hline
\end{tabular}


Table 5. The natural frequencies corresponding to different mass ratios and mass locations for two masses, where $N=100$.

\begin{tabular}{cccccccccccc}
\hline$\alpha_{1}$ & $\alpha_{2}$ & $\eta_{1}$ & $\eta_{2}$ & $\omega_{1}$ & Özk.01 & $\omega_{2}$ & Özk.01 & $\omega_{3}$ & Özk.01 & $\omega_{4}$ & Özk.01 \\
\hline 1 & 1 & 0.1 & 0.3 & 6.118 & 6.118 & 27.536 & 26.506 & 55.338 & 55.412 & 98.966 & 99.097 \\
& & & 0.7 & 6.183 & 6.183 & 22.588 & 22.598 & 60.165 & 60.226 & 124.852 & 125.021 \\
& & 0.5 & 0.3 & 4.730 & 4.785 & 25.116 & 19.802 & 60.830 & 45.252 & 141.073 & 95.238 \\
& & & 0.7 & 4.730 & 4.730 & 25.116 & 25.128 & 60.830 & 60.883 & 141.073 & 141.289 \\
1 & \multirow{2}{*}{10} & 0.1 & 0.3 & 2.509 & 2.509 & 26.066 & 26.075 & 50.993 & 51.069 & 94.388 & 94.505 \\
& & & 0.7 & 2.516 & 2.516 & 20.052 & 20.060 & 58.763 & 58.824 & 124.117 & 124.285 \\
& & 0.5 & 0.7 & 2.387 & 2.387 & 17.916 & 17.925 & 59.518 & 59.569 & 136.776 & 136.993 \\
10 & \multirow{2}{*}{1} & 0.1 & 0.3 & 4.513 & 4.514 & 18.548 & 18.563 & 38.536 & 38.578 & 96.578 & 96.694 \\
& & & 0.7 & 4.671 & 4.671 & 12.423 & 12.429 & 50.941 & 50.992 & 121.270 & 121.432 \\
& & 0.5 & 0.7 & 2.078 & 2.078 & 22.025 & 22.036 & 54.599 & 54.647 & 140.648 & 140.866 \\
& \multirow{2}{*}{10} & 0.1 & 0.3 & 2.356 & 2.357 & 16.238 & 16.257 & 29.949 & 29.975 & 92.758 & 92.863 \\
& & & 0.7 & 2.412 & 2.413 & 8.845 & 8.850 & 48.883 & 48.883 & 120.858 & 121.018 \\
& & 0.5 & 0.7 & 1.677 & 1.677 & 9.806 & 9.812 & 53.472 & 53.516 & 136.317 & 136.535 \\
\hline
\end{tabular}

Table 6. The natural frequencies corresponding to different mass ratios and mass locations for three masses, where $N=100$.

\begin{tabular}{cccccccccccccc}
\hline$\alpha_{1}$ & $\alpha_{2}$ & $\alpha_{3}$ & $\eta_{1}$ & $\eta_{2}$ & $\eta_{3}$ & $\omega_{1}$ & Özk.01 & $\omega_{2}$ & Özk.01 & $\omega_{3}$ & Özk.01 & $\omega_{4}$ & Özk.01 \\
\hline 1 & 1 & 1 & 0.1 & 0.4 & 0.8 & 5.130 & 5.130 & 18.908 & 18.915 & 40.627 & 40.668 & 101.805 & 101.949 \\
1 & 1 & 10 & 0.1 & 0.4 & 0.8 & 3.011 & 3.011 & 11.726 & 11.731 & 39.407 & 39.445 & 98.570 & 98.713 \\
1 & 10 & 1 & 0.1 & 0.4 & 0.8 & 2.182 & 2.182 & 17.179 & 17.186 & 37.318 & 37.356 & 99.182 & 99.323 \\
10 & 1 & 1 & 0.1 & 0.4 & 0.8 & 4.141 & 4.142 & 13.012 & 13.021 & 25.934 & 25.958 & 99.303 & 99.439 \\
10 & 10 & 10 & 0.1 & 0.4 & 0.8 & 1.864 & 1.864 & 6.672 & 6.675 & 14.144 & 14.161 & 93.644 & 93.774 \\
1 & 1 & 1 & 0.2 & 0.5 & 0.7 & 4.411 & 4.411 & 18.193 & 18.201 & 39.151 & 39.189 & 137.782 & 137.980 \\
1 & 1 & 10 & 0.2 & 0.5 & 0.7 & 2.350 & 2.350 & 13.463 & 13.469 & 34.966 & 35.001 & 134.570 & 134.770 \\
1 & 10 & 1 & 0.2 & 0.5 & 0.7 & 2.048 & 2.048 & 18.178 & 18.185 & 29.348 & 29.378 & 137.760 & 137.958 \\
10 & 1 & 1 & 0.2 & 0.5 & 0.7 & 2.857 & 2.857 & 10.767 & 10.771 & 35.346 & 35.379 & 137.078 & 137.274 \\
10 & 10 & 10 & 0.2 & 0.5 & 0.7 & 1.540 & 1.540 & 6.381 & 6.383 & 13.564 & 13.578 & 134.055 & 134.252 \\
\hline
\end{tabular}

As shown in Table 4, the numerical results obtained for the natural frequencies are extremely close to analytical results. Similar findings are presented in Table 5 for two concentrated masses and in Table 6 for three masses. 


\section{CONCLUSIONS}

In this study, a general model is considered to analyze the dynamic behavior of structural elements that may have variable material, cross-section, or some other discontinuities. Unlike the classical approach, instead of writing a separate equation for each span containing discontinuity, a single equation with singularity function is discussed. This provides great convenience to us in the solution, as all discontinuities occurring in any structural element are modeled with a single equation. To demonstrate the accuracy of the present technique, the general solution procedure has been applied to two different problems, such as the multilinear elastic spring beam and beam-mass system. As a result of the comparisons made, it has been observed that the results obtained as a result of applying the classical approach and the present method are extremely close to each other.

\section{ACKNOWLEDGMENT}

This work is supported by Manisa Celal Bayar University Scientific Research Project Coordination Unit (Project number: 2016-113).

\section{REFERENCES}

Beam deflections by discontinuity functions, IAST, Lect12.

Caddemi, S. \& Morassi, A. 2013. Multi-cracked Euler-Bernoulli beams: mathematical modeling and exact solutions. International Journal of Solids and Structures, 50: 944-956.

Caddemi, S., Caliò, I. \& Cannizzaro, F. 2013a. Closed-form solutions for stepped Timoshenko beams with internal singularities and along-axis external supports. Arch. Appl. Mech. 83: 559-577.

Caddemi, S., Caliò, I., Cannizzaro, F. \& Rapicavoli, D. 2013b. A novel beam finite element with singularities for the dynamic analysis of discontinuous frames. Arch. Appl. Mech. 83: 1451-1468.

Cheng, P., Davila, C. \& Hou, G. 2014. Static, vibration analysis and sensitivity analysis of stepped beams using singularity functions. Journal of Structures, 2014(15): 1-13.

Dinev, D. 2012. Analytical solution of beam on elastic foundation by singularity functions. Engineering Mechanics, 19(5): 1-12.

Ghayesh, M.H., Kazemirad, S. \& Reid, T. 2012. Nonlinear vibrations and stability of parametrically exited systems with cubic nonlinearities and internal boundary conditions: A general solution procedure. Applied Mathematical Modelling, 36(7): 3299-3311.

Hosseini, S.A.A. \& Zamanian, M. 2013. Analytical solution for general nonlinear continuous systems in a complex form. Applied Mathematical Modelling, 37(3): 1163-1169.

Lacarbonara, W. 1999. Direct treatment and discretizations of non-linear spatially continuous systems. Journal of Sound and Vibration, 221(5): 849-866.

Nayfeh, A.H. 1981. Introduction to Perturbation Techniques. Wiley, New York.

Sınır, B.G. \& Sınır, S. 2011. Eksenel parametrik zorlamalı çok yaylı kirişlerin yeni bir yaklaşımla dinamik analizi, XVII. National Mechanical Congress, Furat University, Elazı̆g, Turkey.

Sınır, B.G. 2015. Infinite mode analysis of a general model with external harmonic excitation. Applied Mathematical Modelling, 39: $1823-1836$.

Özkaya, E., Pakdemirli, M. \& Öz, H.R. 1997. Non-Linear vibrations of a beam-mass system under different boundary conditions. Journal of Sound and Vibration, 199(4): 679-696.

Özkaya, E. 2001. Linear transverse vibrations of a simply supported beam carrying concentrated mass. Mathematical and Computational Applications, 6(3): 147-151. 